\title{
Magnetically Separable Nanozeolites: Promising Candidates for Bio-Applications
}

Wei Shan, Tao Yu, Bo Wang, Junkai Hu, Yahong Zhang, Xiaoyan Wang, and Yi Tang*

Department of Chemistry and Shanghai Key Laboratory of Molecular Catalysis and Innovative Materials, Fudan University, Shanghai 200433, P.R. China

\section{Supporting Information}

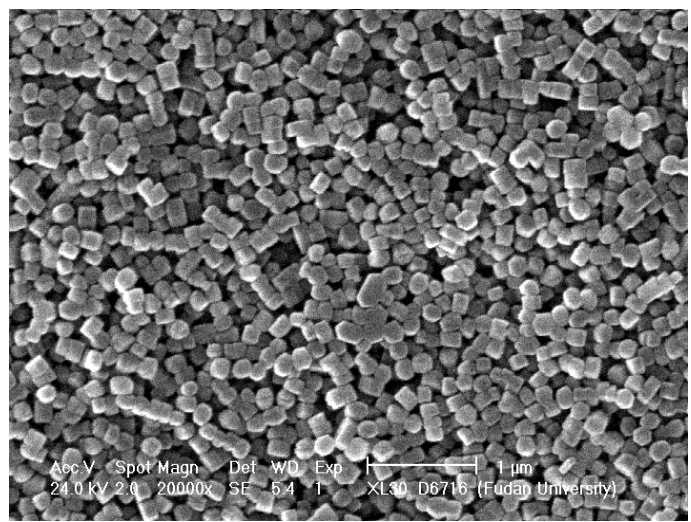

Figure S1. SEM image of nanosized zeolite single crystals, which were obtained under the similar synthesis conditions to MZCNPs except that MNPs were not added in the precursor solution.

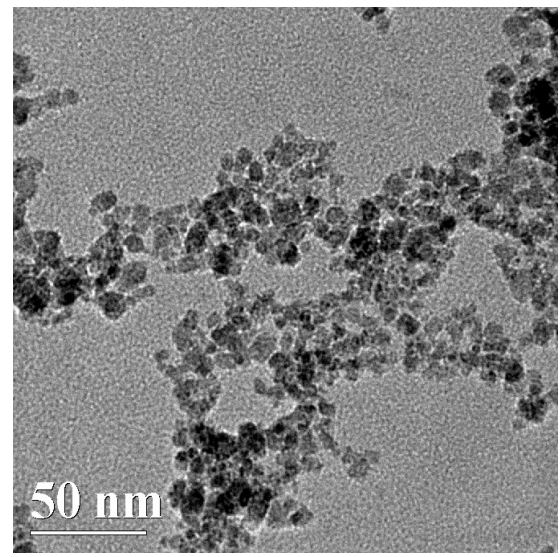

Figure S2. TEM image of MNPs.

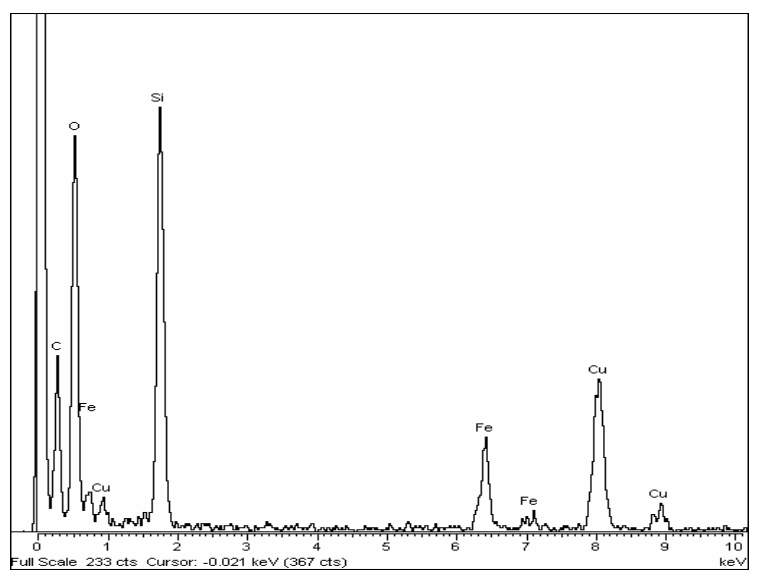

Figure S3. Energy dispersive X-ray (EDX) analysis (equipped on TEM) of the high-contrast dots on MZCNPs. 


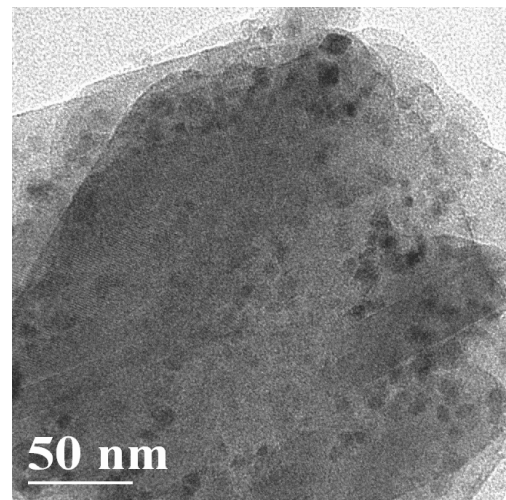

(a)

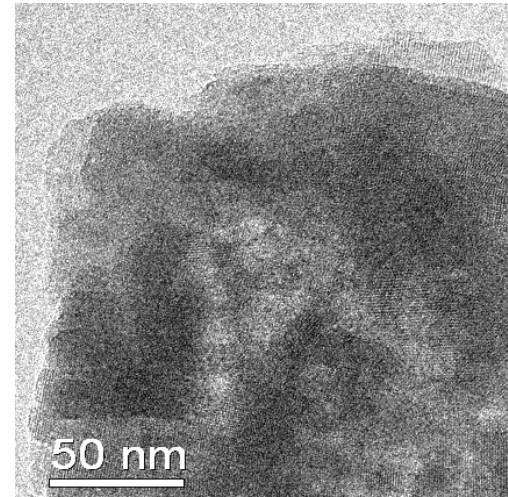

(b)

Figure S4. Comparison of TEM images of MZCNP before (a) and after (b) being treated with concentrated hydrochloric acid (36 wt $\%$ ). The high-contrast dots (MNPs) on MZCNP disappeared after the acid treatment and some low-contrast areas were observed.

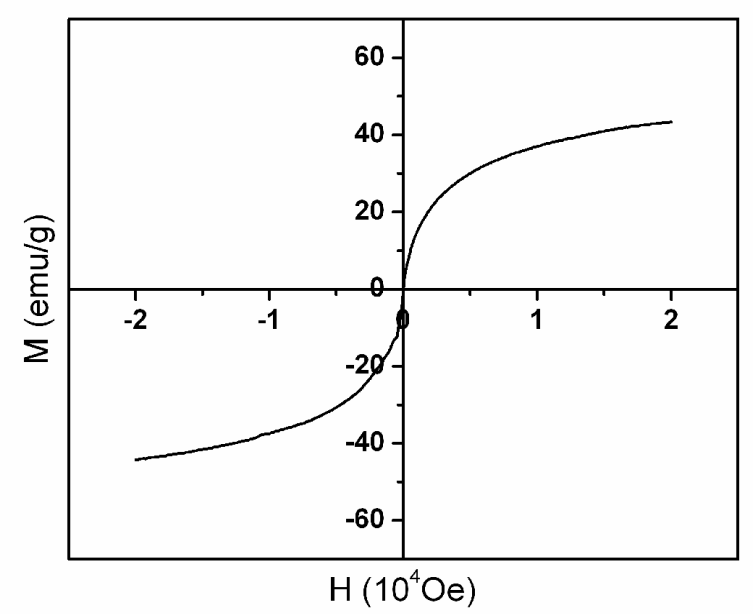

Figure S5. Magnetization curve of MNP at $300 \mathrm{~K}$.

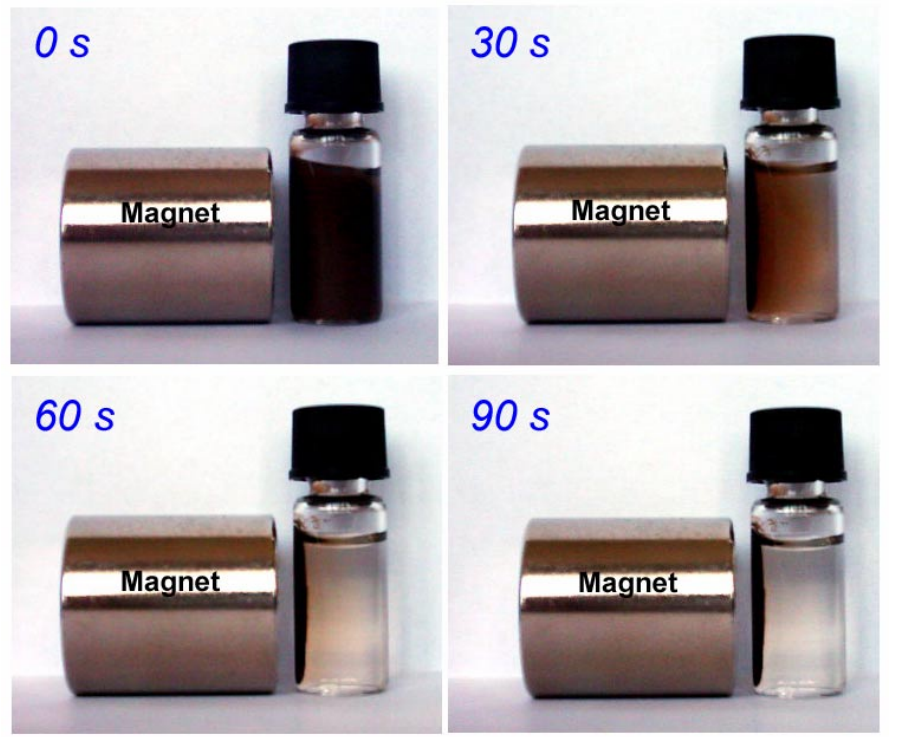

Figure S6. Removal of MZCNPs from their ethanol suspension by a magnet. 


\section{Experimental Section of Biocatalysis}

Reagents. Hemoglobin (from rat) was purchased from Sigma and used without further purification. A phosphate buffer solution (PBS, 25 mmol L-1 pH 7.0) was prepared with $\mathrm{Na}_{2} \mathrm{HPO}_{4}$ and $\mathrm{NaH}_{2} \mathrm{PO}_{4}$. All solutions were prepared with deionized water purified with a Milli Q plus system.

Apparatus. The absorbance of solution was measured on a Shimadzu UV-2450 UV-Vis spectrophotometer. The electrochemical experiments were performed at room temperature in a three-electrode system consisting of a saturated calomel reference electrode (SCE), a platinum wire counter electrode, and an indium tin oxide (ITO) working electrode. All electrochemical measurements were performed on a CHI660b electrochemical working station.

\section{1) Measurement of adsorption amount of hemoglobin on MZCNPs}

$12.5 \mu \mathrm{L}$ of MZCNP suspension $\left(10 \mathrm{mg} \mathrm{mL}^{-1}\right)$ was added to $500 \mu \mathrm{L}$ PBS of hemoglobin $(0.1 \mathrm{mg}$ $\mathrm{mL}^{-1}$ ). After adsorption at room temperature for two hours, the hemoglobin-immobilized MZCNPs (H-MZCNPs) were attracted to the bottom by a magnet. By measuring the UV absorbance at $\lambda=406 \mathrm{~nm}$ of the supernatant solution, the adsorption amount of hemoglobin on MZCNPs was determined (standard curve method).

\section{2) Catalytic activity test of $\mathrm{H}-M Z C N P s$ in the decomposition of $\mathrm{H}_{2} \mathrm{O}_{2}$}

Enzyme immobilization. $200 \mu \mathrm{L}$ of MZCNP suspension $\left(20 \mathrm{mg} \mathrm{mL}^{-1}\right)$ was added into $4 \mathrm{~mL}$ PBS of hemoglobin $\left(0.1 \mathrm{mg} \mathrm{mL}^{-1}\right)$. The adsorption procedure went on at room temperature for four hours. The H-MZCNPs were washed with deionized water and finally dispersed in $200 \mu \mathrm{L}$ of PBS.

Activity test. The H-MZCNP suspension was mixed with $100 \mu \mathrm{L}$ of $\mathrm{H}_{2} \mathrm{O}_{2}$ solution $(30 \mathrm{wt} \%)$ and $700 \mu \mathrm{L}$ of PBS. After $10 \mathrm{~min}$, the H-MZCNPs were attracted to the bottom by a magnet. $100 \mu \mathrm{L}$ of the supernatant solution was diluted to $3 \mathrm{~mL}$ by PBS for UV analysis. Other supernatant solution was removed and the H-MZCNPs were redispersed in $200 \mu \mathrm{L}$ of PBS. The whole procedure was repeated for four times. The catalytic activity of $\mathrm{H}-\mathrm{MZCNPs}$ (the conversion of $\mathrm{H}_{2} \mathrm{O}_{2}$ ) was calculated from the UV absorbance at $\lambda=240 \mathrm{~nm}$ of solution before and after reaction. 


\section{3) Amperometric response to the decomposition reaction of $\mathrm{H}_{2} \mathrm{O}_{2}$ catalyzed by $\mathrm{H}-\mathrm{MZCNPs}$}

Enzyme immobilization. $400 \mu \mathrm{L}$ of MZCNP suspension $\left(6 \mathrm{mg} \mathrm{mL}^{-1}\right)$ was added into $4 \mathrm{~mL}$ PBS of hemoglobin $\left(0.1 \mathrm{mg} \mathrm{mL}^{-1}\right)$. The adsorption procedure went on at $277 \mathrm{~K}$ overnight.

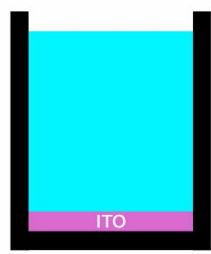

(a)

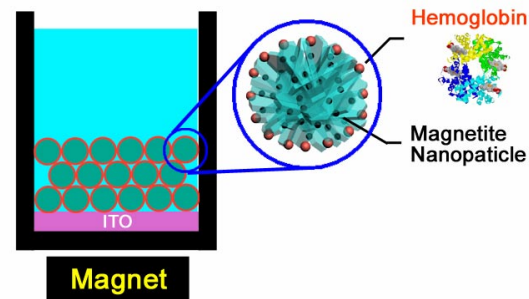

(b)

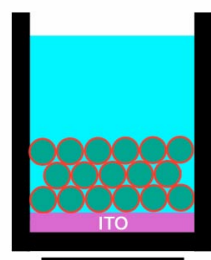

Magnet

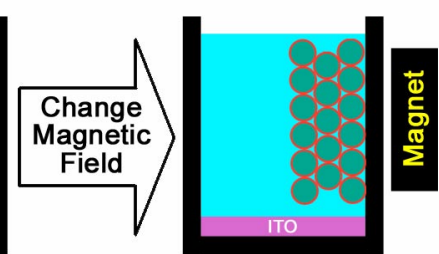

(c)

Figure S7. Schematic illustration of the different surface conditions of ITO electrode in the amperometric measurements: (a) blank experiment, (b) biocatalytic reaction, and (c) quenched biocatalytic reaction, which correspond to the green, red and blue lines in Figure $4 \mathrm{~b}$ (in manuscript), respectively.

Amperometric measurements. In the blank experiment (Figure S7a), the amperometric response of the ITO electrode was measured at $-250 \mathrm{mV}$ in an electrochemical glass cell containing $6 \mathrm{~mL}$ of PBS. When the electrode reached a steady state, $1 \mu \mathrm{L}$ of $\mathrm{H}_{2} \mathrm{O}_{2}$ solution ( $30 \mathrm{wt} \%$ ) was added to the system and the amperometric response was recorded until there was no amperometric change. In the experiment of biocatalytic reaction (Figure S7b), the H-MZCNPs were added and attracted onto the surface of ITO electrode by a magnet. Other experimental conditions were the same as the blank experiment. To further test the manipulability of H-MZCNPs in bio-applications, a quenching experiment was done (Figure S7c). When the current reached the maximum value during the biocatalytic reaction, the orientation of magnetic field was changed to remove the H-MZCNPs from the surface of ITO electrode. 